\title{
ARrioo Concordância do registro informatizado de imunização de Araraquara, São Paulo, 2018*
}

doi: 10.1590/\$1679-49742021000200023

\author{
Computerized immunization record agreement in Araraquara, São Paulo, Brazil, 2018
}

Concordancia del registro de vacunación computarizado de Araraquara, São Paulo, Brasil, 2018

\author{
Érica Marvila Garcia ${ }^{1}$ - (1) orcid.org/0000-0001-9477-2383 \\ Jose Murakami Junior' ${ }^{1}$ - (1) orcid.org/0000-0002-6507-5957 \\ Ângela Aparecida Costa ${ }^{1}$ - (1) orcid.org/0000-0001-5121-3899 \\ Marta Inenami' - (1) orcid.org/0000-0001-8136-0662 \\ Walter Manso Figueiredo' - (1) orcid.org/0000-0003-0772-0089 \\ Eliseu Alves Waldman ${ }^{1}$ - (1) orcid.org/0000-0001-7807-6898 \\ Ana Paula Sayuri Sato' - (1) orcid.org/0000-0001-8601-5884 \\ Universidade de São Paulo, Faculdade de Saúde Pública, São Paulo, SP, Brasil
}

\section{Resumo}

Objetivo: Descrever a concordância entre os dados de imunização do Sistema Juarez e as informações da caderneta de vacinação e as coberturas vacinais em crianças de 12 a 24 meses. Métodos: Estudo descritivo, para avaliar a situação vacinal aos 12 e 24 meses de idade de crianças nascidas em 2015 e registradas no Sistema Juarez. Foram verificados os níveis de concordância entre os dados do Sistema Juarez e as informações da caderneta de vacinação. Resultados: Foram incluídas 429 crianças. Verificou-se que a concordância variou entre 84,1 e 99,1\%. As coberturas no inquérito vacinal para cada vacina variaram de 86,1 a 100\%; e para o esquema completo, de 77,1 (12 meses) a 68,8\% (24 meses). As distribuições espaciais da cobertura vacinal foram de 28 a 100\%. Conclusão: Observou-se ótima concordância entre os dados, com altas coberturas vacinais, muito embora heterogeneidade em suas distribuições espaciais.

Palavras-chave: Vacinação; Programas de Imunização; Cobertura Vacinal; Sistemas de Informação; Análise Espacial.

\footnotetext{
*Artigo derivado da tese de doutorado intitulada 'Hesitação materna em vacinar e a situação vacinal de crianças de até dois anos de idade', a ser defendida por Érica Marvila Garcia junto ao programa de Pós-Graduação em Saúde Pública da Faculdade de Saúde Pública da Universidade de São Paulo, em 2021. Trabalho financiado pela Fundação de Amparo à Pesquisa do Estado de São Paulo (Fapesp): Processo n 2017/14415-9.
}

Endereço para correspondência:

Érica Marvila Garcia - Universidade de São Paulo, Faculdade de Saúde Pública, Av. Dr. Arnaldo, n 715, São Paulo, SP, Brasil. CEP: 01246-904

E-mail: ericamarvila@usp.br 


\section{Introdução}

No contexto histórico, com o prestígio de haver salvado milhares de vidas, a vacinação em massa foi e é considerada uma das grandes conquistas da saúde pública, desde pesquisas fundamentais desenvolvidas em fins do século XIX, até os estudos e a implementação de programas de imunização nacionais nos dias atuais. ${ }^{1}$ Não obstante as evidências de anos de altas coberturas vacinais (>95\%), em 2016, o Brasil apresentou um declínio vacinal de aproximadamente $10 \mathrm{a}$

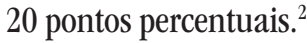

\section{No contexto histórico, com o prestígio de haver salvado milhares de vidas, a vacinação em massa foi e é considerada uma das grandes conquistas da saúde pública.}

As estimativas da cobertura vacinal são geralmente efetuadas pelo método indireto ou administrativo: toma-se para (i) o numerador, os dados agregados, com o total de doses aplicadas ou distribuídas, e para (ii) o denominador, uma estimativa populacional. Este método é útil para o planejamento das ações dos programas de imunização, porém bastante frágil em termos de acurácia. ${ }^{3}$ Da mesma forma que os inquéritos de cobertura vacinal, os registros informatizados de imunização permitem obter dados individuais da população vacinada e identificar microáreas de baixa cobertura, ou seja, bolsões de suscetíveis. ${ }^{3,4}$

0 uso desse instrumento é antigo. Canadá, Reino Unido e Estados Unidos possuem registros informatizados de imunização desde a década de $1970 .{ }^{5}$ A partir de 2006, países da América Latina iniciaram a implantação de registros informatizados de imunização de abrangência nacional para aprimorar as estimativas das coberturas vacinais; à exceção do México e do Uruguai, que utilizam tal instrumento desde 1987 e 1991 respectivamente. $^{6}$

No Brasil, os registros informatizados do Programa Nacional de Imunizações (PNI), reunidos pelo Sistema de Informação do PNI (SI-PNI), vêm-se desenvolvendo desde a década de 1990. Apenas a partir da década de
2010, o SI-PNI vem sendo implantado em todo o território nacional. ${ }^{7}$

Anteriormente à consecução do SI-PNI, algumas iniciativas municipais já existiam no país, a exemplo do Serviço Especial de Saúde de Araraquara (SESA), da Universidade de São Paulo (USP). Município do interior do estado de São Paulo, Araraquara conta com os registros de imunização informatizados desde 1987. Hoje denominado Sistema Juarez, o SESA é, provavelmente, o mais antigo serviço desse caráter no Brasil. ${ }^{5}$ Alguns estudos já foram realizados utilizando dados do Sistema Juarez, ${ }^{8-10}$ entretanto seu potencial para analisar a distribuição espacial das coberturas vacinais, bem como a validação desse sistema em relação aos dados constantes nas cadernetas de vacinação, ainda não foi explorado.

0 objetivo desse artigo foi descrever a concordância entre os dados de imunização do Sistema Juarez e as informações da caderneta de vacinação e as coberturas vacinais em crianças aos 12 e aos 24 meses.

\section{Métodos}

Trata-se de estudo descritivo, com análise de dados secundários do Sistema Juarez e dados primários do inquérito domiciliar realizado no município de Araraquara, SP, em 2018.

Araraquara apresentava 236.072 habitantes em 2019, com aproximadamente 3 mil nascidos vivos por ano e taxa de mortalidade infantil de 10,46 óbitos por 1 mil nascidos vivos; ${ }^{11}$ seu índice de desenvolvimento humano municipal (IDH) é de $0,815^{12}$ e $97,2 \%$ da população está situada na área urbana. ${ }^{13} 0$ município possuía, em 2020, 34 unidades de saúde, três hospitais gerais, dois prontos-socorros, um hospital psiquiátrico e um ambulatório de especialidades, com cerca de $60 \%$ da população local sob cobertura. 0 município também conta com um programa de vacinação identificado por altas coberturas vacinais, refletidas no controle das doenças imunopreveníveis desde a década de $1990 .^{14}$

Localizado no município, o SESA/USP tem seus registros de imunização informatizados desde 1987, quando já contava com 0 registro vacinal individualizado da criança, nome completo do pai e da mãe, nome e data de aplicação da vacina, eventos adversos pós-vacinação, entre outras informações, tendo-se aprimorado nesse sentido, ao longo do tempo. 
Em 2011, o registro de imunização informatizado implementou um prontuário eletrônico de saúde municipal que inclui, além do registro nominal de vacina, dados de consultas e registro de doenças de notificação compulsória. Naquele mesmo ano, o SESA passou a se chamar Sistema Juarez, tornou-se online a partir de 2012 e, desde então, todas as unidades de saúde acessam e registram dados de vacinação em tempo real, no sistema, cuja cobertura é de 99,6\% das crianças nascidas no município. ${ }^{9}$ Pelo Sistema Juarez, são verificados os esquemas de vacinação dos indivíduos nele cadastrados, com data e lote de cada dose de vacina administrada.

0 objeto do estudo constituiu-se de crianças nascidas e residentes em Araraquara no ano de 2015, cadastradas no Sistema Juarez. Foram excluídas as crianças cujas mães não apresentaram caderneta de vacinação da criança durante 0 inquérito vacinal, realizado com amostra probalilística extraída do Sistema Juarez mediante sorteio sem reposição. Cada uma das 15 áreas de ponderação do Instituto Brasileiro de Geografia e Estatística (IBGE) foi considerada um estrato da amostra: $:{ }^{15}$ houve coleta de dados em todos os 15 estratos, mediante entrevistas com as mães de 3.394 crianças nascidas naquele ano e residentes no município, proporcionalmente distribuídas entre as áreas ponderadas pelo IBGE.

Para o cálculo do tamanho amostral mínimo, considerou-se: nível de confiança de $95 \%$, cujo valor foi de 1,96 para um alfa de 0,05; frequência da cobertura vacinal de $40 \%$, com base no estudo de Ferreira et al. ${ }^{9}$ que analisou a cobertura vacinal de 49.741 crianças menores de 2 anos, nascidas de 1998 a 2013 em Araraquara; e erro máximo em valor absoluto de $0,05 .{ }^{16}$ Assim, obteve-se um tamanho amostral mínimo de 369 crianças.

De um total de 3.394 crianças cadastradas no Sistema Juarez em 2015, 3.054 tiveram seus endereços geocodificados e categorizados em suas respectivas áreas de ponderação do IBGE, ${ }^{15}$ de maneira a compor a população final para a amostra aleatória (Figura 1). A partir dessa população final, foram sorteadas 450 crianças; foram acrescentados $20 \%$ do tamanho mínimo amostral calculado, para compensar possíveis perdas.

$\mathrm{Na}$ avaliação da completude do esquema vacinal, foram contempladas as seguintes recomendações de esquema vacinal pelo PNI: ${ }^{17}$ a) Esquema vacinal completo aos 12 meses de idade - uma dose da vacina com o bacilo de Calmette-Guérin (BCG);

- três doses da vacina de difteria, tétano e coqueluche (DPT), Haemophilus influenzae tipo B (Hib) e hepatite B (HEP B) (pentavalente);

- três doses da vacina de poliomielite inativada (VIP);

- duas doses e um reforço da vacina pneumocócica 10 valente;

- duas doses da vacina de rotavírus humano;

- duas doses e um reforço da vacina meningocócica C (conjugada);

- uma dose da vacina de febre amarela; $\mathrm{e}$

- uma dose da vacina de sarampo, caxumba e rubéola (SCR) - tríplice viral.

b) Esquema vacinal completo aos 24 meses de idade - esquema vacinal completo aos 12 meses e reforço da vacina DTP;

- reforço da vacina de poliomielite atenuada (VOP);

- uma dose da vacina de hepatite A; $\mathrm{e}$

- uma dose da vacina tetraviral - SCR e varicela.

Foram consideradas como doses válidas aquelas administradas respeitando-se as idades, mínima e máxima, de aplicação da dose, assim como o intervalo adequado entre as doses para vacinas com esquema multidose (Figura 2).

A coleta de dados do inquérito vacinal aconteceu no período de agosto a outubro de 2018, mediante questionário aplicado em domicílio, por 10 entrevistadores de campo e quatro supervisores, os quais foram submetidos a um treinamento teórico-prático com carga horária de 10 horas, distribuídas em dois dias consecutivos. Previamente, realizou-se um estudo-piloto para adequação do instrumento, envolvendo 20 mães do município de Araraquara não incluídas no estudo. Os dados secundários foram obtidos do Sistema Juarez.

Foram verificados os níveis de concordância entre os dados de vacinação do Sistema Juarez e as informações da caderneta de vacinação coletadas no inquérito vacinal, pelo teste de Kappa, categorizado da seguinte forma: concordância quase perfeita quando 0 coeficiente Kappa foi $\geq 0,80$; concordância substancial, 0,60 a 0,79 ; concordância moderada, 0,41 a 0,59 ; concordância regular, 0,21 a 0,40; e concordância ruim, quando o coeficiente Kappa foi $<0,20$. 


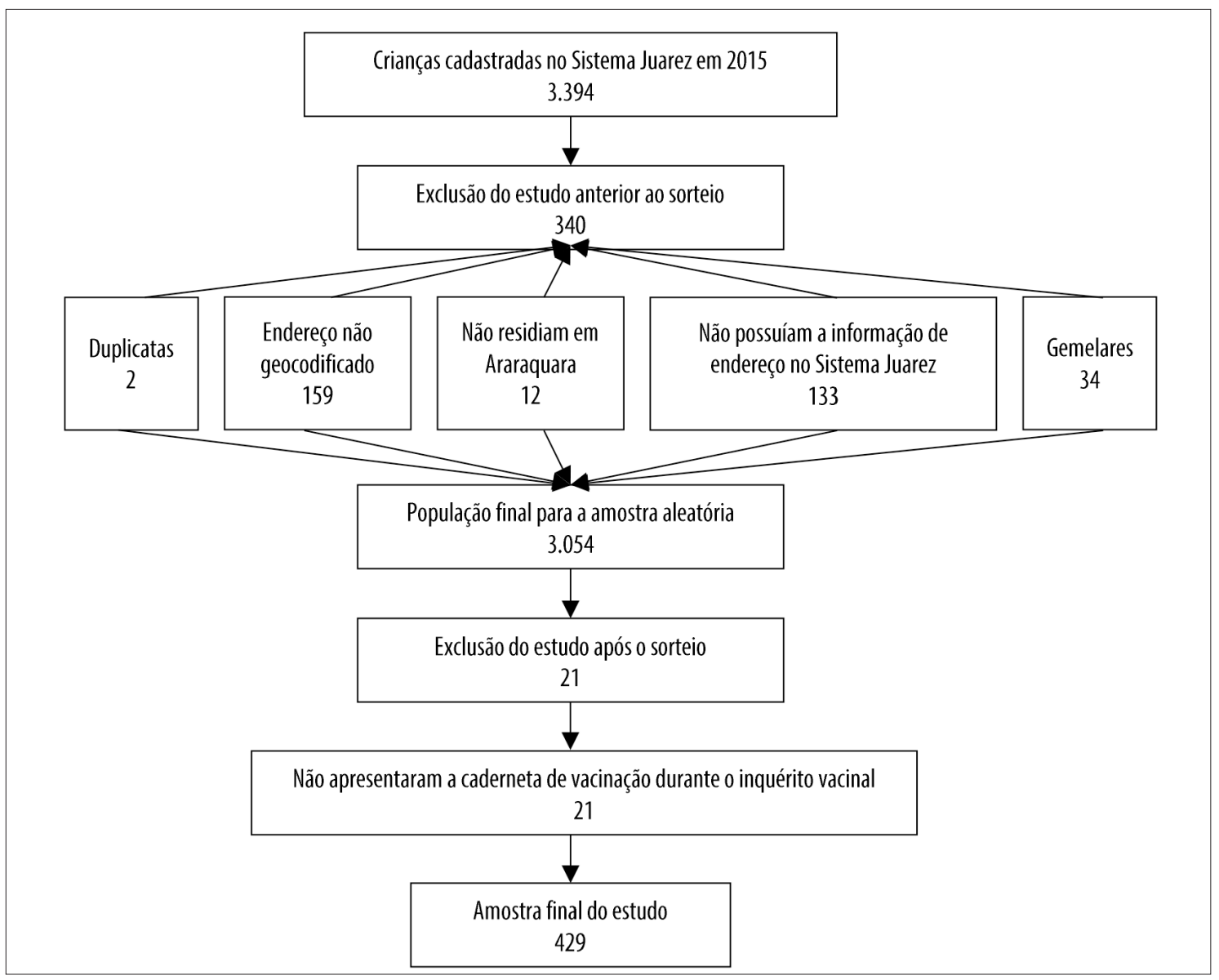

Figura 1 - Processo de recrutamento e exclusão das crianças menores de 2 anos de idade, Araraquara, São Paulo, 2018

0 Sistema de Informação Geográfica foi utilizado para a descrição espacial dos dados de coberturas vacinais, calculadas para cada uma das 15 áreas de ponderação do município e utilizadas no desenho de mapas com sua distribuição. Para tanto, empregou-se o software QGIS 3.4.1 sobre base cartográfica do IBGE. ${ }^{18}$

0 estudo em tela faz parte de um projeto maior, denominado 'Hesitação materna em vacinar e a situação vacinal de crianças de até 2 anos de idade', realizado em 2018. Ele foi novamente aprovado, desta vez pelo Comitê de Ética em Pesquisa da Faculdade de Saúde Pública da Universidade de São Paulo (CEP/FSP/USP): Parecer $n^{0}$ 3.617.912, de 3 de outubro de 2019; Certificado de Apresentação para Apreciação Ética (CAAE) $\mathrm{n}^{0}$ 20721819.0.0000.5421. Foram incluídas no estudo apenas as crianças cujas mães assinaram o Termo de Consentimento Livre e Esclarecido antes do início da entrevista-base do inquérito vacinal.

\section{Resultados}

Do total de 450 crianças admitidas no estudo, 4,7\% foram excluídas: 429 crianças compuseram a amostra final (Figura 1).

Verificou-se concordância entre as duas fontes de dados, em proporção variável de 84,1 a 99,1\%. Houve mais doses registradas no cartão de vacinas do que no Sistema Juarez, sendo essa diferença menor que $5 \%$ para a maioria das vacinas $(74,1 \%)$ (Tabela 1$)$.

0 município de Araraquara apresentou coberturas vacinais de 86,1 a $100 \%$. Ao se considerar o esquema completo, a cobertura da completude vacinal aos 12 meses de idade foi de $77,1 \%$, enquanto para a completude vacinal aos 24 meses de idade, essa cobertura foi de $68,8 \%$ (Figura 3).

As distribuições espaciais das coberturas vacinais não exibiram um padrão definido, com uma cobertura vacinal heterogênea: variação de 25,7 a $100 \%$. 
Figura 2 - Critérios de avaliação de idade mínima e preconizada e intervalos entre doses de vacinas para crianças até 24 meses de idade, segundo o calendário vigente, Araraquara, São Paulo, 2015

\begin{tabular}{|c|c|c|c|c|c|}
\hline Vacina & Dose & Idade mínima & $\begin{array}{c}\text { Intervalo até a } \\
\text { próxima dose }\end{array}$ & $\begin{array}{l}\text { Intervalo mínimo até } \\
\text { a próxima dose }\end{array}$ & $\begin{array}{c}\text { Atraso } \\
\text { (a partir do) }\end{array}$ \\
\hline Bacilo de Calmette-Guérin & 1 & Ao nascer & - & - & $2^{\circ}$ mês \\
\hline \multirow{4}{*}{$\begin{array}{l}\text { Poliomielite atenuada/ } \\
\text { poliomielite inativada }\end{array}$} & 1 & 6 semanas & 2 meses & 4 semanas & $3^{0}$ mês \\
\hline & 2 & 10 semanas & 2 meses & 4 semanas & $5^{0}$ mês \\
\hline & 3 & 14 semanas & 9 meses & 6 meses & $7^{0}$ mês \\
\hline & Reforço & 6 meses após a 3a dose & - & - & $16^{\circ}$ mês \\
\hline \multirow{4}{*}{ Difteria, tétano e coqueluche } & 1 & 6 semanas & 2 meses & 4 semanas & $3^{0}$ mês \\
\hline & 2 & 10 semanas & 2 meses & 4 semanas & $5^{0}$ mês \\
\hline & 3 & 14 semanas & 9 meses & 6 meses & $7^{0}$ mês \\
\hline & Reforço & 12 meses & - & - & $16^{\circ}$ mês \\
\hline \multirow{3}{*}{ Haemophilus influenzae tipo B } & 1 & 6 semanas & 2 meses & 4 semanas & $3^{0}$ mês \\
\hline & 2 & 10 semanas & 2 meses & 4 semanas & $5^{0}$ mês \\
\hline & 3 & 14 semanas & - & - & $7^{0}$ mês \\
\hline \multirow{3}{*}{ Hepatite B } & 1 & Ao nascer & 2 meses & 4 semanas & $2^{\circ}$ mês \\
\hline & 2 & 4 semanas & 4 meses & 8 semanas & $3^{0}$ mês \\
\hline & 3 & 24 semanas & - & - & $7^{0}$ mês \\
\hline \multirow{2}{*}{ Rotavírus humano } & 1 & 6 semanas & 2 meses & 4 semanas & $3^{0}$ mês \\
\hline & 2 & 10 semanas & - & - & $5^{0}$ mês \\
\hline Febre amarela & 1 & 9 meses & - & - & $10^{\circ}$ mês \\
\hline Tríplice viral & 1 & 12 meses & - & - & $13^{\circ}$ mês \\
\hline \multirow{3}{*}{ Pneumocócica 10 valente ${ }^{\mathrm{a}}$} & 1 & 6 semanas & 2 meses & 4 semanas & $3^{0}$ mês \\
\hline & 2 & 10 semanas & 2 meses & 4 semanas & $5^{0}$ mês \\
\hline & Reforço & 12 meses & - & - & $16^{\circ}$ mês \\
\hline \multirow{3}{*}{ Meningocócica C } & 1 & 6 semanas & 2 meses & 4 semanas & $4^{0}$ mês \\
\hline & 2 & 10 semanas & 7 meses & 8 semanas & $6^{0}$ mês \\
\hline & Reforço & 12 meses & - & - & $13^{\circ}$ mês \\
\hline Hepatite A & 1 & 12 meses & - & - & $16^{\circ}$ mês \\
\hline
\end{tabular}

a) Até 2015, eram recomendadas 3 doses da vacina Pneumocócica 10 valente antes dos 12 meses de idade, entretanto, em 2016, houve uma mudança no calendário vacinal e passou-se a orientar a aplicação de apenas duas doses antes dos 12 meses de idade ( $1^{\mathrm{a}} \mathrm{e} 2^{\mathrm{a}}$ dose, respectivamente).

Fonte: Adaptado de Ferreira et al. ${ }^{9} \mathrm{e}$ Tauil et al. ${ }^{10}$

Além disso, a maioria das áreas de ponderação apresentou uma cobertura vacinal acima de $78 \%$ aos 12 meses; 0 que não se repetiu para 0 esquema aos 24 meses, quando essa cobertura se restringiu a apenas quatro áreas (Figura 4).

\section{Discussão}

Houve concordância entre o Sistema Juarez e os registros das cadernetas de vacinação; e altas coberturas vacinais, principalmente das vacinas aplicadas até os
12 meses de idade. As distribuições espaciais das coberturas vacinais não apresentaram um padrão definido; de fato, essa distribuição foi heterogênea, tanto para os esquemas de 12 meses quanto para os de 24 meses.

0 uso de dados secundários pode ser considerado uma limitação do estudo, dada a possibilidade de apresentar incompletude e/ou inconsistências nos registros. Entretanto, ao se utilizar esses dados juntamente com os dados primários do inquérito vacinal, foi possível analisar a qualidade do registro 
Tabela 1 - Número de crianças vacinadas e concordância entre cadernetas de vacinação e Sistema Juarez ( $n=429)$, Araraquara, São Paulo, 2015

\begin{tabular}{|c|c|c|c|c|c|c|}
\hline Vacina & $\begin{array}{c}\text { Caderneta de } \\
\text { vacinação }\end{array}$ & $\begin{array}{c}\text { Sistema } \\
\text { Juarez }\end{array}$ & Diferença (\%) & Concordância (\%) & $\begin{array}{c}\text { Coeficiente } \\
\text { Kappa }\end{array}$ & $\mathrm{p}$-valor ${ }^{\mathrm{a}}$ \\
\hline$B C G^{b}$ & 428 & 429 & $-0,2$ & 97,9 & 0,9 & $<0,001$ \\
\hline Hepatite B ao nascer & 429 & 427 & 0,5 & 97,4 & 0,9 & $<0,001$ \\
\hline DTPc/Hib $/$ hepatite $B-1^{a}$ dose & 429 & 427 & 0,5 & 98,1 & 0,9 & $<0,001$ \\
\hline DTPc/Hib/hepatite B - 2a dose & 428 & 418 & 2,3 & 95,6 & 0,9 & $<0,001$ \\
\hline DTPc/Hib $/$ hepatite B - $3^{a}$ dose & 426 & 422 & 0,9 & 94,1 & 0,9 & $<0,001$ \\
\hline DTPC - Reforço & 415 & 406 & 2,1 & 92,2 & 0,9 & $<0,001$ \\
\hline Poliomielite - $1^{\mathrm{a}}$ dose & 429 & 426 & 0,7 & 99,1 & 0,9 & $<0,001$ \\
\hline Poliomielite $-2^{\mathrm{a}}$ dose & 428 & 423 & 1,2 & 97,2 & 0,9 & $<0,001$ \\
\hline Poliomielite $-3^{\text {a }}$ dose & 426 & 420 & 1,4 & 94,8 & 0,9 & $<0,001$ \\
\hline Poliomielite - Reforço & 417 & 487 & $-16,3$ & 92,4 & 0,9 & $<0,001$ \\
\hline Rotavírus - $1^{\text {a }}$ dose & 424 & 421 & 0,7 & 97,8 & 0,9 & $<0,001$ \\
\hline Rotavírus $-2^{\text {a }}$ dose & 414 & 411 & 0,7 & 96,6 & 0,9 & $<0,001$ \\
\hline Pneumococo - $1^{\text {a }}$ dose & 429 & 425 & 0,9 & 97,9 & 0,9 & $<0,001$ \\
\hline Pneumococo $-2^{\text {a }}$ dose & 426 & 423 & 0,7 & 97,2 & 0,9 & $<0,001$ \\
\hline Pneumococo - reforço & 424 & 391 & 7,7 & 84,1 & 0,8 & $<0,001$ \\
\hline Meningococo $-1^{\text {a }}$ dose & 425 & 424 & 0,2 & 95,5 & 0,9 & $<0,001$ \\
\hline Meningococo $-2^{\text {a }}$ dose & 422 & 422 & 0,0 & 92,8 & 0,9 & $<0,001$ \\
\hline Meningococo - Reforço & 413 & 404 & 2,1 & 94,6 & 0,9 & $<0,001$ \\
\hline Febre amarela & 423 & 414 & 2,1 & 93,9 & 0,9 & $<0,001$ \\
\hline Hepatite A & 427 & 409 & 4,2 & 93,6 & 0,9 & $<0,001$ \\
\hline Sarampo/caxumba/rubéola - 1a dose & 427 & 414 & 3,0 & 94,7 & 0,9 & $<0,001$ \\
\hline Sarampo/caxumba/rubéola - 2a dose & 354 & 381 & $-6,3$ & 92,6 & 0,9 & $<0,001$ \\
\hline Varicela & 393 & 379 & 3,3 & 95,7 & 0,9 & $<0,001$ \\
\hline
\end{tabular}

a) p-valor do teste de Kappa; b) BCG: bacilo de Calmette-Guérin; c) DTP: difteria, tétano e coqueluche; d) Hib: haemophilus influenzae tipo B.

informatizado de imunizações, estruturado e consolidado. A perda amostral, que também poderia representar uma limitação para o estudo, não só foi pequena como se manteve dentro da margem aceitável, prevista no cálculo amostral, sem poder de interferência nos resultados obtidos.

A alta cobertura vacinal encontrada também foi descrita em outros estudos, sobre nascidos em Araraquara nos anos de 1998 e 2013. ${ }^{9,10}$ Tal cobertura pode estar relacionada à organização do programa municipal de imunizações e ao bom desempenho do Sistema Juarez, sob contínua melhoria ao longo do tempo; e ao uso de ferramentas eficientes no monitoramento das coberturas vacinais, caso do relatório de faltosos/atrasados (recall), que permite a busca ativa para atualização de esquemas vacinais, e do relatório de crianças com vacinas agendadas (remind), para aumento da adesão e aproveitamento da oportunidade da vacinação. ${ }^{9}$

Assim como os resultados do presente estudo, 0 inquérito vacinal brasileiro de 2007, conduzido nas 26 capitais de estados e no Distrito Federal, mostrou que $82,6 \%$ das crianças receberam todas as vacinas até os 18 meses de idade. ${ }^{19}$ Outras pesquisas, como a realizada em São Luís, capital do estado do Maranhão, em 2006, por meio de um inquérito domiciliar, encontrou uma cobertura vacinal aos 12 meses de idade de $71,9 \% ;{ }^{20}$ em Pelotas, RS, dados da coorte de nascidos vivos de 2015 evidenciaram cobertura de 77,0\%. ${ }^{21}$ Esses estudos reforçam a presença de boas coberturas vacinais. 


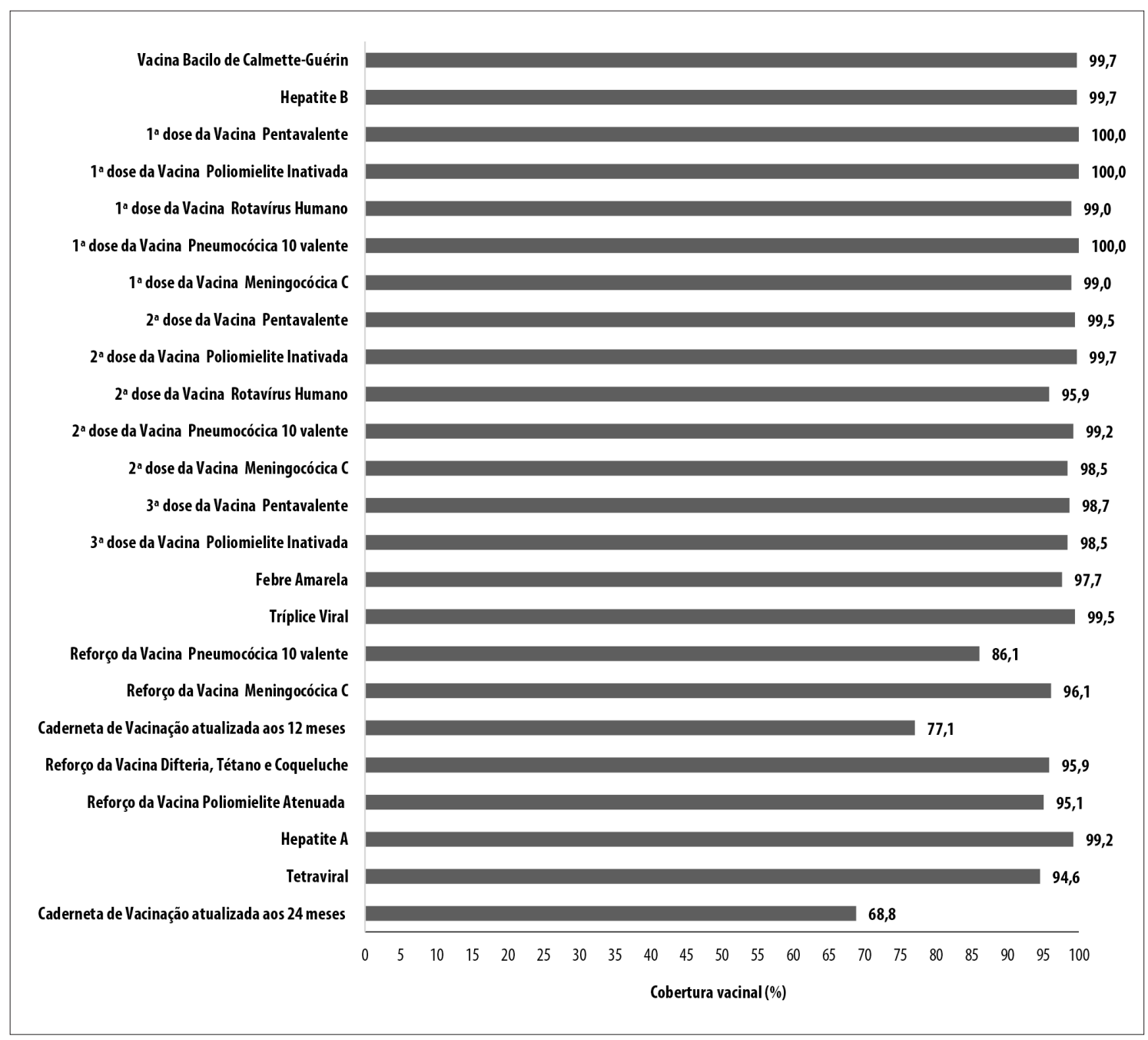

Figura 3 - Coberturas vacinais atualizadas por dose de vacinas e esquema completo aos 12 e aos 24 meses de idade (n=429), Araraquara, São Paulo, 2018

A distribuição espacial permite visualizar a heterogeneidade das coberturas vacinais entre as áreas de ponderação e, por conseguinte, o monitoramento das coberturas vacinais das microáreas, um aspecto fundamental para o sucesso do PNI. Esse método tem sido cada vez mais utilizado na área da Saúde, tendo contribuído não só para a melhoria das atividades de vigilância mas, também para a identificação de áreas de risco, merecedoras de intensificação ou priorização de medidas de cobertura vacinal. ${ }^{22,23}$

Ainda sobre a cobertura vacinal, a heterogeneidade observada também foi relatada em pesquisa realizada no estado do Ceará, onde, apesar das altas coberturas vacinais contra o sarampo, quatro das 21 coordenadorias regionais de saúde não alcançaram a meta de 95\% ${ }^{24}$ Uma pesquisa dedicada à análise de uma série histórica (2013 a 2017) de coberturas vacinais em estado fronteiriço da Amazônia brasileira, sobre dados disponibilizados no Sistema de Informação do PNI, evidenciou a dificuldade em manter coberturas vacinais homogêneas em escala nacional, dadas as baixas coberturas vacinais de algumas regiões do país. ${ }^{25}$ Tais resultados podem se explicar pelas características do território brasileiro, sua grande diversidade social e cultural, e a influência de fatores sociodemográficos, como emprego/ocupação - especialmente entre as mães -, 


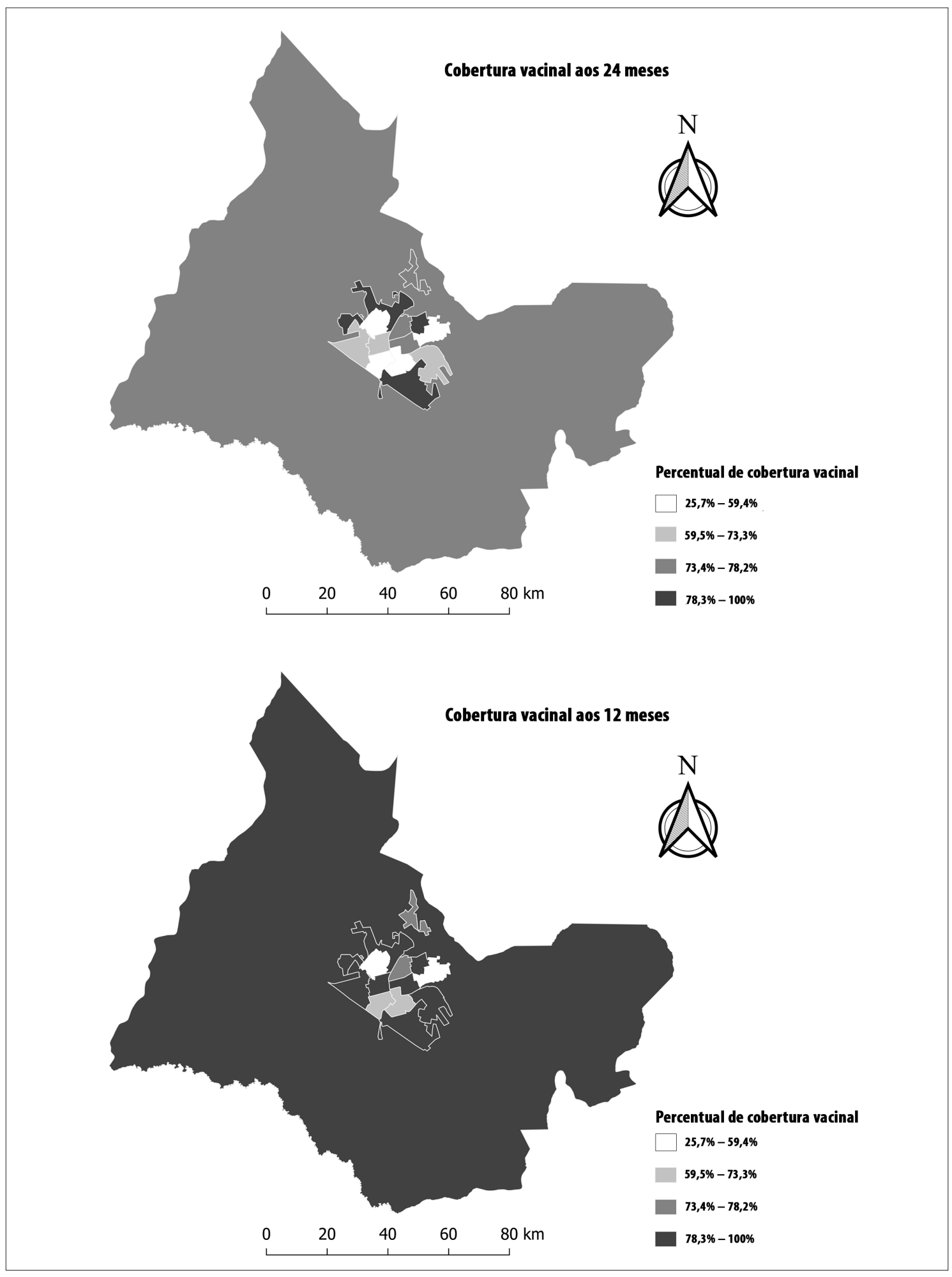

a) IBGE: Instituto Brasileiro de Geografia e Estatística.

Figura 4 - Distribuição espacial das coberturas da completude vacinal aos 12 e aos 24 meses de idade, segundo área de ponderação do IBGEa ( $n=429$ ), Araraquara, São Paulo, 2018 
renda familiar, escolaridade pública das crianças, baixa escolaridade de seus responsáveis, número elevado de filhos por mãe e ordem de nascimento dessas crianças, compreensão das razões e da importância da vacinação, confiança no sistema público de saúde, e outros igualmente determinantes, como a distância física e 0 acesso a cuidados de saúde, ademais de questões programáticas, como o fornecimento de insumos e vacinas pela rede de frios. ${ }^{26,27}$

A ótima concordância observada entre o Sistema Juarez e a caderneta de vacinação da criança demonstra que em Araraquara, o registro informatizado dos dados de imunização apresenta uma cobertura vacinal de elevada acurácia. 0 achado corrobora 0 entendimento de que a experiência pioneira do SESA no desenvolvimento e uso do Sistema Juarez, ao longo de mais de três décadas, foi tanto exitosa quanto contribuiu para a qualidade do PNI e sua boa cobertura vacinal no município paulista. A eficiência no uso dos registros informatizados de imunização, ademais de favorecer 0 aumento das coberturas vacinais, conforme já foi relatado ${ }^{28}$ reafirma a importância da precisão e da representatividade dos registros dos dados da população, possíveis de serem utilizados no planejamento das ações. ${ }^{29}$

Os resultados do presente estudo avalizam-no a orientar políticas públicas para o resto do país e servir de subsídio ao Sistema de Informação do Programa Nacional de Imunizações (SI-PNI). Eles evidenciam 0 potencial dos registros informatizados na ampliação da cobertura e qualificação dos programas de imunizações em curso, enquanto uma ferramenta eficiente de monitoramento e avaliação das coberturas vacinais, proporcionando a identificação de microáreas de baixa cobertura vacinal e bolsões de suscetíveis a doenças imunopreveníveis.

Conclui-se que o Sistema Juarez é um registro informatizado de imunização bastante confiável, nos dados que apresenta, útil ao monitoramento e vigilância das coberturas vacinais. Araraquara, outrossim, conta com uma boa cobertura vacinal, embora sua distribuição espacial tenha-se revelado heterogênea.

\section{Contribuição dos autores}

GarciaEMeSatoAPS contribuíram,substancialmente, para a concepção do estudo, análise e interpretação dos dados, elaboração das versões preliminares e versão final do manuscrito. Murakami-Junior J, Costa AA, Inenami M, Figueiredo WM e Waldman E contribuíram com a análise e interpretação dos dados e revisão crítica do conteúdo intelectual do manuscrito. Todos os autores aprovaram a versão final e são responsáveis por todos os aspectos do trabalho, incluindo a garantia de sua precisão e integridade.

\section{Referências}

1. Dubé E, Laberge C, Guay M, Bramadat P, Roy $\mathrm{R}$, Bettinger J. Vaccine hesitancy: an overview. Hum Vaccin Immunother. 2013;9(8):176373. doi: https://doi.org/10.4161/hv.24657.

2. Sato APS. Qual a importância da hesitação vacinal na queda das coberturas vacinais no Brasil? Rev Saude Publica. 2018;52(96):1-9. doi: http://doi. org/10.11606/s1518-8787.2018052001199.

3. Cutts FT, Claquin P, Danovaro-holliday MC, Rhoda DA. Monitoring vaccination coverage: Defining the role of surveys. Vaccine. $2016 \mathrm{Jul}$ 19;34(35):4103-9. doi: http://dx.doi.org/10.1016/j.vaccine.2016.06.053.

4. Sato APS. Programa nacional de imunização: sistema informatizado como opção a novos desafios. Rev Saude Publica. 2015;49(39):1-5. doi: http:// doi.org/10.1590/S0034-8910.2015049005925.
5. Luhm KR, Waldman EA. Sistemas informatizados de registro de imunização: uma revisão com enfoque na saúde infantil. Epidemiol Serv Saude. 2009;18(1):65-78. doi: http://dx.doi. org/10.5123/S1679-49742009000100007.

6. Danovaro-holliday MC, Ortiz C, Cochi S, Ruizmatus $\mathrm{C}$. Electronic immunization registries in Latin America: progress and lessons learned. Rev Panam Salud Publica. 2014;35(5-6):453-7.

7. Domingues CMAS, Teixeira AMS. Coberturas vacinais e doenças imunopreveníveis no Brasil no período 1982-2012: avanços e desafios do programa nacional de imunizações. Epidemiol Serv Saude. 2013;22(1):9-27. doi: http://dx.doi. org/10.5123/S1679-49742013000100002. 
8. Sato APS, Ferreira VLR, Tauil MC, Rodrigues LC, Barros $\mathrm{MB}$, Martineli E, et al. Uso de registro informatizado de imunização na vigilância de eventos adversos pós-vacina. Rev Saude Publica. 2018;52(4):1-10. doi: https://doi.org/10.11606/S1518-8787.2018052000295.

9. Ferreira VLR, Waldman EA, Rodrigues LC, Martineli E, Costa ÂA, Inenami M, et al. Avaliação de coberturas vacinais de crianças em uma cidade de médio porte (Brasil) utilizando registro informatizado de imunização. Cad Saude Publica. 2018;34(9):1-11. doi: https://doi.org/10.1590/0102-311x00184317.

10. Tauil MC, Sato APS, Costa AA, Inenami M, Ferreira VLR, Waldman EA. Coberturas vacinais por doses recebidas e oportunas com base em um registro informatizado de imunização, Araraquara-SP, Brasil, 2012-2014. Epidemiol Serv Saude. 2017;26(4):835-46. doi: https:// doi.org/10.5123/s1679-49742017000400014.

11. Instituto Brasileiro de Geografia e Estatística. Taxa de mortalidade infantil [Internet]. 2017 [acesso 5 nov. 2020]. Disponível em: https://cidades.ibge.gov.br/brasil/ sp/araraquara/pesquisa/39/30279?tipo=ranking

12. Atlas do Desenvolvimento Humano no Brasil. Unidades de desenvolvimento humano [Internet]. 2020 [acesso 14 maio 2020]. Disponível em: http:// www.atlasbrasil.org.br/2013/pt/o_atlas/metodologia/ construcao-das-unidades-de-desenvolvimento-humano/

13. Instituto Brasileiro de Geografia e Estatística. População [Internet]. 2019 [acesso 5 nov. 2020]. Disponível em: https://cidades.ibge. gov.br/brasil/sp/araraquara/panorama

14. Departamento de informática do SUS. Informações de saúde [Internet]. 2020 [acesso 14 maio 2020]. Disponível em: http:/www2.datasus.gov.br/ DATASUS/index.php?area $=0203 \& i d=29878153$

15. Instituto Brasileiro de Geografia e Estatística. Notas metodologicas [Internet]. 2011 [acesso 14 maio 2020]. Disponível em: https://www.ibge.gov.br/ apps/snig/v1/notas_metodologicas.html?loc $=0$

16. Berquó E, Souza JMP, Gotlieb SLD. Bioestatística. São Paulo: EPU; 1981.460 p.

17. Ministério da Saúde (BR). Calendário nacional de vacinação [Internet]. 2019 [acesso 20 mar. 2020]. Disponível em: https://antigo.saude.gov.br/ saude-de-a-z/vacinacao/calendario-vacinacao\# ${ }^{\#}$ crianca

18. Instituto Brasileiro de Geografia e Estatística. Mapas, bases e referenciais, bases cartográficas, malhas digitais [Internet]. 2019 [acesso 13 jul. 2020]. Disponível em: https://mapas.ibge.gov.br/bases-e-referenciais/ bases-cartograficas/malhas-digitais

19. Barata RB, Ribeiro MCSA, Moraes JC, Flannery B, Group behalf of the VCS 2007. Socioeconomic inequalities and vaccination coverage: results of an immunisation coverage survey in 27 Brazilian capitals, 2007-2008. J Epidemiol Community Health. 2012;66(10):934-41. doi: http://dx.doi.org/ 10.1136/jech-2011-200341

20. Yokokura AVCP, Silva AAM, Bernardes ACF, Lamy Filho F, Alves MTSSB, Cabral NAL, et al. Cobertura vacinal e fatores associados a esquema vacinal básico incompleto aos 12 meses de idade, São Luís, Maranhão, Brasil, 2006. Cad Saude Publica. 2013;29(3):522-34. doi: https://doi. org/10.1590/S0102-311X2013000300010.

21. Buffarini R, Barros FC, Silveira MF. Vaccine coverage within the first year of life and associated factors with incomplete immunization in a Brazilian birth cohort. Arch Public Health. 2020 Apr 8;78:21. doi: https://doi. org/10.1186/s13690-020-00403-4. eCollection 2020.

22. Arroyo LH, Ramos ACV, Yamamura M, Weiller TH, Crispim JA, Cartagena-Ramos D, et al. Áreas com queda da cobertura vacinal para BCG, poliomielite e tríplice viral no Brasil (2006-2016): mapas da heterogeneidade regional. Cad Saude Publica. 2020;36(4):e00015619. doi: http://dx.doi.org/10.1590/0102-311X000155619.

23. Galli B, Chiaravalloti Neto F. Modelo de risco tempo-espacial para identificação de áreas de risco para ocorrência de dengue. Rev Saude Publica. 2008;42(4):656-63. doi: http://dx.doi. org/10.1590/S0034-89102008005000032.

24. Moura ADA, Braga AVL, Carneiro AKB, Alves ECS, Bastos CMM, Nunes IH, et al. Monitoramento rápido de vacinação na prevenção do sarampo no estado do Ceará, em 2015. Epidemiol Serv Saude. 2018;27(2): e2016380. doi: http://dx.doi. org/10.5123/s1679-49742018000200017.

25. Zambonin F, Lima KLB, Sousa PAC, Muniz TR, Caldart RV, Maciel JC, et al. Análise da cobertura vacinal em menores de cinco anos em um estado fronteiriço da Amazônia. Saude Redes [Internet]. 2019;5(2):289-99. doi: https://doi. org/10.18310/2446-48132019v5n2.2240g389.

26. Guzman-Holst A, Deantonio R, Prado-cohrs D, Juliao P. Barriers to vaccination in Latin America: a systematic literature review. Vaccine. 2020 Jan 16;38(3):470-81. doi: https://doi.org/10.1016/j.vaccine.2019.10.088. 
27. Tertuliano GC, Stein AT. Atraso vacinal e seus determinantes: um estudo em localidade atendida pela estratégia saúde da família. Cienc Saude Colet. 2011;16(2):523-30.

28. Groom H, Hopkins DP, Pabst LJ, Morgan JM, Patel $\mathrm{M}$, Calonge N, et al. Immunization information systems to increase vaccination rates: a community guide systematic review. J Public Health Manag Pract. 2015;21(3):227-47. doi: https://doi. org/10.1097/PHH.0000000000000069.

29. Placzek H, Madoff LC. The use of immunization registry-based data in vaccine effectiveness studies. Vaccine. 2011 Jan 10;29(3):399-411. doi: http:// dx.doi.org/10.1016/j.vaccine.2010.11.007.

\section{Abstract}

Objective: To describe agreement between the Juarez System immunization data and information in vaccination record booklets and vaccination coverage in children aged 12 to 24 months. Methods: This was a descriptive study to assess the vaccination status at 12 and 24 months of age of children born in 2015 and recorded on the Juarez System. The levels of agreement between the Juarez System data and the information in vaccination record booklets were verified. Results: 429 children were included. It was found that agreement ranged between $84.1 \%$ and $99.1 \%$. The vaccine survey found that coverage for each vaccine ranged from $86 \%$ to $100 \%$ and for the full schedule, from $77.1 \%$ (12 months) to $68.8 \%$ (24 months). The spatial distributions of vaccine coverage ranged from $28 \%$ to $100 \%$. Conclusion: There was excellent agreement between the data, with bigh vaccination coverage, but heterogeneity in their spatial distributions.

Keywords: Vaccination; Immunization Programs; Vaccination Coverage; Information Systems; Spatial Analysis.

\section{Resumen}

Objetivo: Describir la concordancia entre los datos de vacunación del Sistema Juárez y la información de la cartilla de vacunación y cobertura vacunal en niños de 12 a 24 meses. Métodos: Estudio descriptivo para evaluar el estado de vacunación a los 12 y 24 meses de edad de los niños nacidos en 2015 e inscritos en el Sistema Juárez. Se verificaron los niveles de concordancia entre los datos del Sistema Juárez y la información del carné de vacunación. Resultados: Se incluyeron 429 niños. Se encontró que la concordancia varió entre 84,1 y 99,1\%. La cobertura (encuesta de vacunas) para cada vacuna varió de $86 \%$ a $100 \%$ y para el esquema completo, $77,1 \%$ (12 meses) y 68,8\% (24 meses). Las distribuciones espaciales de la cobertura vacunal oscilaron entre el 28\% y el 100\%. Conclusión: excelente concordancia entre los datos, alta cobertura de vacunación, pero heterogeneidad en sus distribuciones espaciales.

Palabras clave: Vacunación; Programas de Inmunización; Cobertura de Vacunación; Sistemas de Información; Análisis Espacial.

Recebido em 04/10/2020

Aprovado em 13/01/2021 